\title{
Two folk theorems in topological dynamics
}

\author{
Joseph Auslander ${ }^{1}$
}

Received: 13 October 2015 / Revised: 23 January 2016 / Accepted: 26 January 2016 /

Published online: 11 February 2016

(C) Springer International Publishing AG 2016

\begin{abstract}
We present (and prove) two "obvious" theorems in topological dynamics. That is, it would appear that the conclusion follows immediately from the definitions, but in fact this is not the case. While these results are "well known" the author has not seen them in print.
\end{abstract}

Keywords Proximal - Regionally proximal $\cdot$ Distal and equicontinuous structure relations

\section{Mathematics Subject Classification $\quad 54 \mathrm{H} 20$}

A flow $(X, T)$ is a jointly continuous action of the topological group $T$ on the compact Hausdorff space $X,(t, x) \mapsto t x, e x=x$, and $s(t x)=$ stx for $x \in X$, $s, t \in T$.

If $(X, T)$ is a flow and $x \in X$ the orbit of $x$ is the set $T x=\{t x: t \in T\}$, and its closure $\overline{T x}$ is the orbit closure of $x$. A subset of $X$ is said to be invariant if it is a union of orbits.

If $(X, T)$ is a flow, a minimal set is a non-empty, closed, invariant set which is minimal with respect to these properties. Equivalently it is the orbit closure of each of its points. A simple Zorn's Lemma argument shows that minimal sets always exist for flows on a compact space. If $(X, T)$ is itself minimal it is called a minimal flow.

This paper is dedicated to the memory of Robert Ellis, who died in December 2013. Bob Ellis was the leading researcher in the abstract theory of topological dynamics and much of what is in this paper was directly inspired by his work.

$凶$ Joseph Auslander

jna@math.umd.edu

1 Department of Mathematics, University of Maryland, College Park, MD 20742-0001, USA 
If $(X, T)$ and $(Y, T)$ are flows, a homomorphism from $X$ to $Y$ is a continuous surjective map $\pi: X \rightarrow Y$ such that $\pi(t x)=t \pi(x)$ for $t \in T$ and $x \in X$. We say that $Y$ is a factor of $X$, and that $X$ is an extension of $Y$.

The simplest flows are the equicontinuous ones, where the maps defined by the group $T$ form an equicontinuous family. (In the case of a metric space these are characterized by the " $\varepsilon-\delta$ " definition: if $\varepsilon>0$ there is $\delta>0$ such that whenever $d\left(x, x^{\prime}\right)<\delta$ then $d\left(t x, t x^{\prime}\right)<\varepsilon$ for all $t \in T$.)

A more general notion is distality. This is defined in terms of the proximal relation $P$. We say that $x$ and $y$ in $X$ are proximal $((x, y) \in P)$ if for any neighborhood $W$ of the diagonal $\Delta$ there is $t \in T$ such that $(t x, t y) \in W$. In the metric case this says $\inf _{t \in T} d(t x, t y)=0$. If $x$ and $y$ are not proximal, they are said to be distal, and the flow $(X, T)$ is distal if any pair of distinct points are distal. It is easily checked that an equicontinuous flow is distal. An example of a distal flow which is not equicontinuous will be given later (in the discussion of regional proximality).

Equicontinuous and distal flows are reasonably well understood. In both cases they are the (necessarily disjoint) union of minimal sets. Equicontinuous minimal flows are homogeneous spaces of topological groups. The analysis of distal minimal flows was carried out in a deep paper of Hillel Furstenberg [4], where it is shown that such flows can be obtained by a (possibly transfinite) sequence of "equicontinuous extensions" starting with the "trivial" one point flow.

Therefore, given an arbitrary flow $(X, T)$, one might try to "reduce" it to a distal or equicontinuus flow by a homomorphism, or what is the same thing, to find a closed $T$ invariant equivalence relation $R$ so that the factor flow $(X / R, T)$ is distal or equicontinous.

Indeed (considering distality for now) it is easy to see that a flow has a maximal distal factor. Equivalently there is a smallest closed $T$ invariant equivalence relation $S_{\mathrm{d}}$ (the distal structure relation) such that the factor flow $\left(X / S_{\mathrm{d}}, T\right)$ is distal.

This is a consequence of the easily proved facts that an arbitrary product of distal flows is distal, as is a closed invariant subset, and the trivial, one point flow. It follows that if $\left\{R_{i}\right\}$ is the set of closed invariant equivalence relations such that $\left(X / R_{i}, T\right)$ is distal, and $R=\bigcap R_{i}$ then $(X / R, T)$ is the maximal distal factor of $(X, T)$.

Since for any flow homomorphism $\pi: X \rightarrow Y, \pi\left(P_{X}\right) \subset P_{Y}$ it follows that $S_{\mathrm{d}}$ contains $P$ - that is, to obtain a distal flow from a given one, it is necessary to collapse proximal pairs. In general $P$ is not an equivalence relation. For example, this is the case for a weakly mixing minimal flow [1, Chapter 9], so $P$ may be a proper subset of $S_{\mathrm{d}}$.

A natural conjecture is that $S_{\mathrm{d}}$ is the closed $T$ invariant equivalence relation generated by $P$. This is in fact the case, and it is proved in [3]. The proof is by no means trivial. The first of our "folk theorems" is a generalization.

Theorem 1 Let $\pi: X \rightarrow Y$ be a homomorphism offlows such that if $\left(x, x^{\prime}\right) \in P$ then $\pi(x)=\pi\left(x^{\prime}\right)$. Then $Y$ is distal.

Note that if it were the case that whenever $\pi: X \rightarrow Y$ is a flow homomorphism, then $\pi\left(P_{X}\right)=P_{Y}$ then the conclusion would follow immediately. But this is not true in general. Here is a simple counterexample. 
Let $X$ consist of two copies of the unit interval equipped with the map $x \mapsto x^{2}$. Let $Y$ be obtained from $X$ by identifying the endpoints, giving a homeomorphism of the circle (so $T=\mathbb{Z}$ the additive group of integers). Any pair of points from the upper and lower arc are proximal, but clearly their (unique) inverse image consists of points on different intervals so are not proximal.

(If $(Y, T)$ is minimal then it can be shown that $\pi\left(P_{X}\right)=P_{Y}$.)

The proof of Theorem 1 depends on the enveloping semigroup, which is one of Ellis' major contributions.

If $X$ is a compact Hausdorff space, $X^{X}$ consists of the collection of all (not just continuous) maps from $X$ to itself, provided with the topology of pointwise convergence. By Tychonoff's theorem $X^{X}$ is compact. $X^{X}$ is a semigroup under composition of maps. It has very limited continuity properties - if $f_{i}, f, g \in X^{X}$ with $f_{i} \rightarrow f$, then $f_{i} g \rightarrow f g$, and in general that is all one can say.

Now identify the group $T$ with the self maps of $X$ given by its action, so we may regard $T$ as a subset of $X^{X}$. Let $E(X, T)$ (or $E(X)$ or $E$ ) denote the closure of $T$ in $X^{X}$ so $E(X)$ is compact. Moreover $E$ is a subsemigroup of $X^{X}$, and is called the enveloping semigroup of the flow $(X, T)$. The maps in $E$ are not in general continuous or even Borel, and $E$ has the same limited continuity properties as $X^{X}$. (Note that they are limits of nets, not necessarily of sequences.) As if to compensate for this, $E$ has a rich algebraic structure, which can be correlated with the dynamical properties of $(X, T)$.

The enveloping semigroup is particularly useful in studying proximality. Suppose $\left(x, x^{\prime}\right) \in P$. Then there is a net $\left\{t_{i}\right\}$ in $T$ such that $t_{i}\left(x, x^{\prime}\right) \rightarrow \Delta$. Let (a subnet) of $t_{i} \rightarrow \eta \in E$. Then we have $\eta x=\eta x^{\prime}$. A similar argument shows that if $\eta x=\eta x^{\prime}$ for some $\eta \in E$ then $x$ and $x^{\prime}$ are proximal. Therefore $\left(x, x^{\prime}\right) \in P$ if and only if $\eta x=\eta x^{\prime}$ for some $\eta \in E(X)$.

If $\left(x, x^{\prime}\right) \in P$, let $H=\left\{\eta \in E: \eta x=\eta x^{\prime}\right\}$. As we have just seen $H \neq \varnothing$ and it is immediate that $H$ is closed. Moreover, $H$ is a left ideal - if $\lambda \in E$ and $\eta \in H$, then $\lambda \eta \in H$. It can be shown that every non-empty closed left ideal contains a minimal left ideal. So we have $\left(x, x^{\prime}\right) \in P$ if and only if $p x=p x^{\prime}$ for all $p \in I$ for some minimal left ideal $I$ in $E$.

Also, it can be shown by a Zorn's Lemma argument that a minimal left ideal contains idempotents $u \in I$ with $u^{2}=u$. The dynamical significance of this is that if $u$ is an idempotent and $x \in X$ then $x$ and $u x$ are proximal, since $u x=u^{2} x=u(u x)$.

If $(Y, T)$ is a factor of $(X, T), \pi: X \rightarrow Y$ then there is induced a (semigroup) homomorphism $\theta: E(X) \rightarrow E(Y)$ such that $\pi(p x)=\theta(p) \pi(x)$. We suppress $\theta$ notationally and regard $E(X)$ as acting on $Y$ and write $\pi(p x)=p \pi(x)$, for $p \in E(X)$.

Now for the proof of Theorem 1. Suppose $\left(y, y^{\prime}\right) \in P$. Then $p y=p y^{\prime}$ for $p \in I$ a minimal left ideal. In particular $u y=u y^{\prime}$ for $u$ an idempotent in $I$. Now let $\left(x, x^{\prime}\right) \in$ $X \times X$ with $\pi\left(x, x^{\prime}\right)=\left(y, y^{\prime}\right)$. Then $(x, u x) \in P$ so $\pi(x)=\pi(u x)$ and similarly $\pi\left(u x^{\prime}\right)=\pi\left(x^{\prime}\right)$. Then $y=\pi(x)=\pi(u x)=u y=u y^{\prime}=\pi\left(u x^{\prime}\right)=\pi\left(x^{\prime}\right)=y^{\prime}$.

Just as for distal, given a flow $(X, T)$, there is an equicontinuous structure relation, a smallest closed $T$ invariant equivalence relation $S_{\text {eq }}(X)$ such that the factor flow $\left(X / S_{\mathrm{eq}}(X), T\right)$ is equicontinuous, and the proof of the existence of $S_{\mathrm{eq}}(X)$ is completely analogous to the proof for $S_{\mathrm{d}}(X)$ given earlier. 
The key to $S_{\mathrm{eq}}$ is the regionally proximal relation $R P$. The pair $\left(x, x^{\prime}\right) \in R P$ if there are nets $\left\{x_{i}\right\}$ and $\left\{x_{i}^{\prime}\right\}$ in $X$ and $\left\{t_{i}\right\}$ in $T$ with $x_{i} \rightarrow x, x_{i}^{\prime} \rightarrow x^{\prime}$ and $t_{i}\left(x_{i}, x_{i}^{\prime}\right) \rightarrow \Delta$. (If $X$ is a metric space, then $\left(x, x^{\prime}\right) \in R P$ if and only if, given $\varepsilon>0$ there are $z$ and $z^{\prime}$ in $X$ and $t \in T$ such that $d(x, z)<\varepsilon, d\left(x^{\prime}, z^{\prime}\right)<\varepsilon$ and $d\left(t z, t z^{\prime}\right)<\varepsilon$.)

Clearly $P \subset R P$. The relation $R P$ is reflexive, symmetric, $T$ invariant, and closed, but not in general an equivalence relation.

A simple example illustrating regional proximality is provided by the homeomorphism of the disc in polar coordinates $f(r, \theta)=(r, r+\theta)$. The flow generated by $f$ is distal, but $R P$ is not trivial. For example $\left(\left(1, \theta_{1}\right),\left(1, \theta_{2}\right)\right) \in R P$ for all $\theta_{1}, \theta_{2}$.

It is immediate that a flow $(X, T)$ is equicontinuous if and only if $R P=\Delta$.

If $Y$ is a factor of $X$, then $\pi\left(R P_{X}\right) \subset R P_{Y}$, and just as in the case of $S_{\mathrm{d}}$ one expects that $S_{\text {eq }}$ is the smallest closed invariant equivalence relation containing $R P$. Again, this is in fact the case. Its proof, given in [3], depends on a deep theorem of Ellis, namely that a flow is equicontinuous if and only if its enveloping semigroup is a group of homeomorphisms [2, Chapter 4]. (A related theorem, also due to Ellis, which has applications in other branches of analysis, is that a locally compact Hausdorff group for which multiplication is separately continuous is a topological group.)

A generalization is our second folk theorem, concerning equicontinuity.

Theorem 2 Let $\pi: X \rightarrow Y$ be a homomorphism, with $\pi(R P)=\Delta$. Then $(Y, T)$ is equicontinuous.

In fact there is a simple proof of Theorem 2, which does not require the joint continuity theorem.

Let $y_{n} \rightarrow y, t_{n}\left(y, y_{n}\right) \rightarrow\left(y^{\prime}, y^{\prime \prime}\right)$. It is an elementary exercise to check that for equicontinuity of $(Y, T)$ it is sufficient to show that $y^{\prime}=y^{\prime \prime}$. Let $\pi\left(x_{n}\right)=y_{n}, x_{n} \rightarrow x$ so $\pi(x)=y$. Let $t_{n}\left(x, x_{n}\right) \rightarrow\left(x^{\prime}, x^{\prime \prime}\right)$ so $\pi\left(x^{\prime}, x^{\prime \prime}\right)=\left(y^{\prime}, y^{\prime \prime}\right)$. But $\left(x^{\prime}, x^{\prime \prime}\right) \in R P$, $t_{n}^{-1}\left(t_{n} x, t_{n} x_{n}\right)=\left(x, x_{n}\right) \rightarrow(x, x)$, so $\pi\left(x^{\prime}\right)=\pi\left(x^{\prime \prime}\right)$. That is $y^{\prime}=y^{\prime \prime}$.

So, somewhat surprisingly, the proof for equicontinuity and regional proximality is easier than for distality and proximality.

It is natural to consider the relative versions of the above theorems. That is, let $(X, T)$ and $(Y, T)$ be flows and let $\pi: X \rightarrow Y$ be a homomorphism. Let $P_{\pi}$ be the relative proximal relation, $P_{\pi}=\left\{\left(x, x^{\prime}\right): \pi(x)=\pi\left(x^{\prime}\right)\right.$ and $\left.\left(x, x^{\prime}\right) \in P\right\}$. Suppose $(Z, T)$ is a flow, and $\alpha: X \rightarrow Z$ and $\beta: Z \rightarrow Y$ are homomorphisms with $\pi=\beta \alpha$ and $\alpha\left(P_{\pi}\right)=\Delta_{Z}$. (If $Y=1$ the trivial one point flow, this is the situation considered in Theorem 1.) The natural conjecture is that $\beta$ is a distal homomorphism, that is that $P_{\beta}=\Delta$.

However, without some additional assumptions this is not the case. Let $X$, as in the example given earlier, be two copies of $I$ with the homeomorphism $x \mapsto x^{2}$ on each interval. Let $Y=I$ with the same homeomorphism. Let $Z$ be the circle with the induced homeomorphism (called $Y$ in the first example), and let $\alpha: X \rightarrow Z$, $\beta: Z \rightarrow Y, \pi: X \rightarrow Y$ be the obvious homomorphisms. Then $P_{\pi}=\Delta$ so of course $\alpha\left(P_{\pi}\right)=\Delta$ but $\beta$ is not distal, in fact it is proximal.

If we assume that $(Y, T)$ is minimal then $\beta: Z \rightarrow Y$ is a distal homeomorphism. The proof, included for completeness, is almost the same as in the absolute case.

Let $\left(z, z^{\prime}\right) \in P_{\beta}$ and let $y=\beta(z)=\beta\left(z^{\prime}\right)$. Let $I$ be a minimal left ideal for which $p z=p z^{\prime}$ for $p \in I$ and let $u$ be an idempotent in $I$ such that $u y=y$. Let $x, x^{\prime} \in X$ 
with $\alpha\left(x, x^{\prime}\right)=\left(z, z^{\prime}\right)$ (so $\left.\pi(x)=\pi\left(x^{\prime}\right)\right)$. Then $(x, u x) \in P_{\pi}$ so $\alpha(u x)=\alpha(x)$ and similarly $\alpha\left(u x^{\prime}\right)=\alpha\left(x^{\prime}\right)$. Then $z=\alpha(x)=\alpha(u x)=u z=u z^{\prime}=\alpha\left(u x^{\prime}\right)=\alpha\left(x^{\prime}\right)=$ $z^{\prime}$.

Similar considerations apply to the relative regionally proximal relation $R P_{\pi}$, $\left(x, x^{\prime}\right) \in R P_{\pi}$ if there are nets $\left(x_{n}, x_{n}^{\prime}\right) \rightarrow\left(x, x^{\prime}\right)$ with $\pi\left(x_{n}\right)=\pi\left(x_{n}^{\prime}\right)$ (so $\left.\pi(x)=\pi\left(x^{\prime}\right)\right)$ and $t_{n} \in T$ such that $t_{n}\left(x_{n}, x_{n}^{\prime}\right) \rightarrow \Delta$.

It is easily checked that the homomorphism $\pi$ is equicontinuous if and only if $R P_{\pi}=\Delta$. The same counterexample shows that if $\alpha\left(R P_{\pi}\right)=\Delta$ it does not follow that $\beta$ is an equicontinuous extension.

Just as in the case of the relative proximal relation, if $(Y, T)$ is minimal, then $\beta$ is equicontinuous. This follows from a more general result [1]: If $(X, T),(Y, T),(Z, T)$, $\pi, \alpha$, and $\beta$ are as above with $(Y, T)$ minimal, then $\alpha\left(R P_{\pi}\right)=R P_{\beta}$. Therefore if $\alpha\left(R P_{\pi}\right)=\Delta$, then $R P_{\beta}=\Delta$ and $\beta$ is equicontinuous.

In conclusion we would like to call the reader's attention to a recent book [2] of Bob Ellis and his son David, Automorphisms and Equivalence Relations in Topological Dynamics, which develops topological dynamics from the point of view of "ICERS" (invariant closed equivalence relations).

\section{References}

1. Auslander, J.: Minimal Flows and their Extensions. North-Holland Mathematics Studies, vol. 153. North-Holland, Amsterdam (1988)

2. Ellis, D.B., Ellis, R.: Automorphisms and Equivalence Relations in Topological Dynamics. London Mathematical Society Lecture Note Series, vol. 412. Cambridge University Press, Cambridge (2014)

3. Ellis, R., Gottschalk, W.H.: Homomorphisms of transformation groups. Trans. Amer. Math. Soc. 94(2), 258-271 (1960)

4. Furstenberg, H.: The structure of distal flows. Amer. J. Math. 85(3), 477-515 (1963) 